\title{
Proceedings of the First World Congress on Fertility Preservation: executive summary
}

\author{
Jacques Donnez • S. Samuel Kim • David F. Albertini
}

Published online: 13 May 2010

(C) Springer Science+Business Media, LLC 2010

\author{
Abbreviations \\ ART assisted reproductive technology \\ FP fertility preservation \\ CO cryopreservation \\ IVM in vitro maturation \\ OTCP ovarian tissue cryopreservation \\ ISFP international society of fertility preservation
}

The inaugural World Congress on Fertility Preservation was held from December 10-12, 2009 in Brussels under the auspices of the newly formed International Society of Fertility Preservation (ISFP). ISFP President Professor Jacques Donnez organized a three day program bringing together some 35 experts as speakers from around the world. Faculty and session moderators evoked much discussion on a range of topics pertinent to this fast-developing branch of Assisted Reproduction Technologies (ARTs). Over 350 clinicians and scientists from around the world participated. In breaking ground for what is rapidly emerging as an ever-increasing dictum to bring preventive medicine into the forefront of reproductive medicine, this conference served two novel and insightful functions:

To bring focus to the special needs of cancer patients with respect to their prospects for child bearing in the future.

Capsule A summary of the platform contributions at the First International Congress of Fertility Preservation is provided.

\section{J. Donnez}

Catholic University of Louvain,

Brussels, Belgium

S. S. Kim • D. F. Albertini

Kansas University Medical Center,

Kansas City, KS, USA

D. F. Albertini $(\bowtie)$

Kansas University Cancer Center,

3901 Rainbow Boulevard,

Kansas City, KS 66103, USA

e-mail: dalbertini@kumc.edu
To assess the status of clinical and basic science research in human reproduction pertinent to medical or environmental interventions that impact present and future fertility.

The substance of the congress left most in attendance with the view that while challenges remain, prospects were bright for fertility preservation to develop into an important field bringing previously unanticipated benefits for human health in general and infertility management in particular. The meetings overall structure was designed to achieve three goals: Set the stage for the clinical basis of the field, bear witness to the technologies that are under development that or are already making contributions to the treatment of patients, especially those being treated for cancer, and finally, engage the participants in a lively debate on the benefits and risks associated with the available technologies. The learning objectives included:

How to approach fertility preservation in children and adults

Define the impact of cancer treatment on gonads

Review cryopreservation techniques

Introduce the most recent methods being used in FP

Discuss the advantages and disadvantages of fertility preservation strategies using gonadal cells, tissues and whole organs

How to approach fertility preservation in adults and children and defining the impact of cancer treatment on gonads

Professor Wallace (UK) reviewed the practical nature of pediatric oncology in boys and girls and reinforced the notion that offering options to these families provides hope during trying times. He cautioned that reliable studies have been difficult to complete given the high drop out rate for 
families whose children survive. Nevertheless, for young males, sperm banking prior to treatment remains the best approach to fertility preservation except in prepubertal boys where few options exist. For young females, serious challenges are evident since these children are not in a position to choose options available for mature women with partners (embryo freezing, oocyte cryopreservation). In these patients, ovarian tissue cryopreservation (OTCP) is the most promising with 30 cases of auto-transplanted frozen-thawed tissue resulting in the birth of seven live young to date. This approach is appropriate for pre-pubertal girls and precludes the use of ovarian hyperstimulation but suffers from the drawbacks associated with surgical retrieval of the ovary and the risks associated with cancer cell contamination in cases of hematological or other malignancies. The theme of ovarian and uterine damage was further developed by Dr. Meirow (IL). In focusing on ovarian damage, Meirow emphasized that both chemotherapy and radiation in young females lead to the well known consequences of reduced ovarian weight, a decreased number of ovarian follicles and stromal fibrosis with chemotherapy being specifically associated with the depletion of primordial follicles. He emphasized the need for preserving the maximal number of follicles because young girls today are more likely to wait to have children and will require preservation of as many follicles as possible. In this light, the relative impact of various forms of chemotherapy on follicle loss were reviewed with alkylating agents being the most ovotoxic while loss induced by plant alkaloids including taxanes is less severe. While uncovering the basis for ovotoxicity after chemotherapy remains a priority, Dr. Meirow encouraged investigation of technologies that would provide protection to the damaging effects of these agents on the follicle reserve. In this context, he cited recent studies in mice that have identified c-abl as a putative target for limiting chemotherapy induced follicle loss and reported on promising studies ranging from the commonly used GnRH agonists, S1P as a apoptosis regulator, Imantinib a cabl inhibitor, and a technicium compound that acts as an immunomodulator of IL-10. This provocative presentation also called attention to shifting the emphasis from germ cells to the various somatic cells of the ovary as potential targets for harnessing the devastating effects of chemotherapy on ovarian functionality.

Professor Tourmaye (BL) reviewed the impact of chemotherapy and radiation on the testis noting that while Leydig and Sertoli cells are relatively resistant to these treatments, postnatal and pubertal patients may suffer long term consequences due to endocrine or exocrine insufficiency at these critical stages of testicular development. While reduced sperm cell counts or azoospermia result from varying degrees of damage to the spermatogonial stem cell, often culminating from apoptotic cell death elicited in response to DNA damage, the degree of compromised function can vary widely again depending on the type of treatment used. He further emphasized that even with recovery of spermatogenesis, concerns regarding the transmission of chromosomal defects or mutations to offspring have not been eliminated and warrant further study. Finally, prospects for transplantation of spermatogonial stem cells were considered as a future treatment option that will require more basic research to establish its potential suitability.

Dr. Rosendahl emphasized that patient age, chemotherapy dose and type are the most important factors that will contribute to chemotherapy induced amenorrhea and that careful monitoring of ovarian functional biomarkers can in some cases be predictive of post treatment outcome. Reporting on two studies using $\mathrm{AMH}, \mathrm{FSH}$, and antral follicle counts (AFC), the suggestion was made that the higher bioavailability to treatment in young patients may predispose these individuals to an increased risk of amenorrhea relative to an older patient population.

Professor Englert (BL) extended the discussion regarding the utility of GnRH agonists as a protective treatment for ovarian damage during chemotherapy pointing out that neither the mode of action of such treatments nor effectiveness have yet to be confirmed. Interestingly, this paper challenged the notion that apoptosis in oocytes is in fact the driving force in follicle loss after chemotherapy. Rather, a more careful investigation of the mechanisms of cell death that result in ovotocicity was proposed where the emphasis should be placed on the granulose cells that interact with the oocyte especially since loss of primordial follicles is taking place at a FSH independent stage of follicle development.

A recurrent theme during the meeting was the need to identify patient specific needs for fertility preservation depending upon the nature of the malignancy being managed. In the case of breast cancer patients, this was illustrated and analyzed in the lecture by Dr. Dor(IL) as he reviewed the various scenarios he has managed in the context of the stage of disease progression at the time of diagnosis. Whether or not patients present with positive ER/BRCA1 genotypes was weighed in determining the most appropriate course of treatment. In essence, the concerns regarding controlled ovarian hyperstimulation as an additional risk factor in cases of IVF and embryo storage were reviewed in the context of what other alternatives might exist for patients, especially those presenting with suspect genetic predispositions (e.g.BRCA1). In cases where time permit, both ovarian tissue cryopreservation (OTCP) and in vitro maturation of oocytes (IVM) were offered. He emphasized that introducing IVM for this high risk subpopulation was feasible and appropriate since minimal ovarian stimulation was required and oocyte retrieval could be conducted during the luteal phase of the 
cycle. Attending to the particular needs and requirements for each patient was effectively conveyed to the audience further expanding discussion of the risks and limitations of FP options being encountered in the context of age and disease status.

\section{Review cryopreservation techniques and introduce the most recent methods being used in FP}

Low temperature storage of tissues and cells has been a mainstay in the field of FP and much debate has arisen as to the utility and safety of these procedures and the best strategies to employ. Professors Kim (USA), Donnez(BE), and Andersen(DK) addressed the current clinical status of this area with respect to the use of cryobanked ovarian tissues. Kim reported long term restoration of ovarian function (for 6 years) and fertility after heterotopic transplantation of frozen-thawed human ovarian tissue in four women with cancer. He emphasized the role of heterotopic ovarian transplantation. He further discussed the need to solve the problem of tissue ischemia that results after grafting as this, rather than freezing and thawing of tissue, is likely to be the primary cause of graft failure and follicle demise. In summarizing the global experience with orthotopic transplantation of ovarian cortex, Donnez reported that 11 live births have resulted with slow freeze cryopreservation having been used in all cases for tissue storage and that six of the resultant conceptions were achieved naturally whereas five of the cases involved IVF. Andersen described the Danish experience from the perspective of how tissue transportation can be coordinated at a national level to assure that cryopreservation is obtained in a timely and efficient manner. Maximizing the number of tissue pieces that are stored for a given patient was discussed (ideally 15-30) to make possible subsequent transplantations should the initial grafting attempt fail. The topic of whole ovary transplantation was next reviewed in an examination of the results that have been obtained in animal and human studies. Dr. Salle (FR) summarized the many years of experience that his laboratory has had using the sheep as an ovary transplantation model. Despite various efforts to achieve long term functionality, he showed that loss of primordial follicles took place and alluded to the drawbacks that remain following vascular reanastomsis with respect to the extreme sensitivity that follicles exhibit during even transient periods of ischemia. Dr. Patrizio reinforced the concerns over the long term suitability of ovary transplantation and cited the difficulties in attaining successful organ cryopreservation as being a limiting factor. The achievements with human material were reviewed by Dr. Silber who has pioneered efforts in the USA and has met with success using microsurgical approaches that he likened to those being used for skin grafting. As seen throughout the meeting, many questions remain as to the suitability of these approaches and how to best overcome these obstacles may ultimately require adoption of animal models that bring to bear the particular challenges that are currently impeding progress for human applications.

\section{Introduce the most recent methods being used in FP}

Efforts to achieve testicular tissue preservation were reviewed by Dr. Wyns (BE) and despite efforts in this direction, the intrinsic architecture of the testis has precluded major advances in this area indicating that sperm cryopreservation remains the most viable approach in ageappropriate young men. This was discussed in the context of progress being made in animal models where replenishing testicular function by germ cell transplantation via the rete testis has resulted in production of transgenic offspring but serious limitations would impede comparable approaches for fertility restoration in humans.

The next session addressed the use of organ and cell culture techniques to sustain the viability and production of mature oocytes. The overarching goal of these approaches is to avail developmentally competent oocytes as a result of ovarian tissue storage and subsequent use of single or multistep culture models that would support both the growth and maturative stages of oogenesis. Prof. Smitz (BE) introduced the session by reviewing the subject of human folliculogenesis in vivo in the context of what we know about these processes as they occur in vitro (see review Smitz et al. 2010). He raised the key question based on the classical studies of Gougeon suggesting that the available data indicate that the complete process of follicluogenesis in humans is estimated to exceed 100 days and to date all efforts to sustain follicles in culture with viable oocytes have approached a matter of weeks in duration. Moreover, he emphasized the need to define the regulatory factors that control key transitions in folliculogenesis as this knowledge will be central to optimizing in vitro conditions to support both follicle and oocyte development. Finally, emphasis was placed on the events in oocyte development that are associated with the actual process of maturation as sufficient recent information exists to establish this phase of oogenesis as essential for the oocyte to acquire the molecular machinery for fertilization and early embryogenesis. This subject was reinforced by Prof. Albertini (US) who provided a summary of the factors that directly affect oocyte quality. After reviewing recent knowledge regarding the physiology of ovulation in mammals, he proceeded to outline how current conditions of IVM as used for human oocytes compare and contrast to the environmental conditions with the in vivo environment 
of the follicle. New results were presented that emphasize how cytoplasmic maturation is a multifaceted entity involving distinct kinase signaling pathways of the src family tyrosine kinases (see McGinnis and Albertini, JARG, 2010) in the oocyte cortex. Moreover, the importance of maintaining cumulus cell contact throughout IVM was also demonstrated to be a major factor in assuring that oocytes are able to support the events of fertilization and early development.

In drawing attention to the need to develop an artificial ovary as a means to preclude reintroduction of cancer cells embedded within ovarian tissues, Dr. Dolmans (BE) next presented results aimed at addressing this implicit risk associated with ovarian tissue transplantation. Her studies revealed the presence of cancer cell markers in frozenthawed ovarian tissue from both leukemic patients and patients with breast cancer. That this problem could be managed by follicle culture was raised as was the need to introduce an artificial ovary system that would at least be able to minimize, and hopefully eliminate, cancer cell contamination within derivative obvarian tissues (that is follicles) prior to reimplantation into patients. Efforts to reach this goal were then reviewed by Professors Amorin (BE) and Woodruff (US). Amorin considered recent efforts with the use of fibrin clots as a scaffolding for the culture of human preantral follicles emphasizing issues of biomaterials compatibility to sustain progressive development of follicles. In a widely recognized approach, Woodruff summarized her laboratories efforts using the alginate gel system in animal and more recent primate and human studies. This promising alternative for follicle culture has been shown to yield viable oocytes in mice and now the conditions have been suitably modified to sustain both non-human primate and human follicles with viable oocytes contained therein. Attempts to now characterize the quality of these oocytes are ongoing and interesting results were presented using polarization microscopy to monitor the integrity of cumulus cell oocyte interactions during the 6-8 day course of culture.

Prof. Telfer (UK) then addressed closing the gap between in vitro growth (IVG) and IVM based on her laboratories work with bovine and human ovarian cortical tissues that were cultured either as fresh tissue or following cryopreservation and thawing. She emphasized that the ability to achieve nearly full growth of oocytes over a 10 20 day culture period is both practical and viable when sequential conditions are implemented to meet the changing needs of the follicle during first the activation of primordial follicles and subsequently during the transition to secondary multilayered stages. These prospects were further evidenced from the work of Prof. Hovatta (SE) who reported on the importance of long-recognized factors such as GDF9, insulin, and IFG-like molecules to engage and regulate the pace of both follicle activation and later growth under conditions conducive for the propagation of healthy oocytes. While the ultimate goals of achieving IVM and IVF for these oocytes remains to be achieved, sufficient progress in this area was provided to catalyze further studies to improve conditions for IVG and IVM of human ovarian follicles.

Dr. Gook (AUS) discussed the impact of cryopreservation and grafting on folliculogenesis using immunodeficient mice as recipicients for human ovarian tissue xenografts. She too emphasized that while cryopreservation alone does not result in serious tissue damage, the major problem of tissue ischemia following grafting has been difficult to avoid and/or minimize given the high incidence of stromal cell apoptosis evident within 2 days of grafting. Whether addition of angiogenesis promoting factors will exacerbate these deleterious effects remains to be demonstrated but represents a promising direction for future studies. This session ended with a presentation by Prof. S L Tan (CAN) who has been adopting human IVM and oocyte vitrification as a course of management for cancer patients. Noting that IVM is an effective treatment option for infertile patients, having resulted in the birth of over 1,000 healthy children globally, he encouraged further use of IVM for storage of mature oocytes by vitirification to address the growing needs of patients who for various reasons would not be suitable candidates for traditional IVF cycles.

The Keynote lecture for the meeting was given by Prof. Gosden who has been widely and appropriately credited for having set the stage for this field based on his many important and insightful contributions over the past 20 years. Prof. Gosden treated the audience with his typically thoughtful and provocative account of where the field of stem cell research may or may not play into the current discourse of novel approaches to fertility preservation. His historical account of the topic of stem cells in the ovary brought perspective on the origins of the debate and great difficulties that attended these discussions due to technical limitations and interpretations engendered in past and present methods for quantifying follicle number. After reviewing the more recent work in mice of the possibility of ovarian stem cells regenerating new follicles and oocytes, he left the audience on a somewhat skeptical note regarding the potential applications for endogenous ovarian stem cells or the more recently proposed induced pluripotent stem cells (iPS cells) that have garnered much attention of late for various uses in regenerative medicine.

\section{Discuss the advantages and disadvantages of fertility preservation strategies using gonadal cells, tissues and whole organs}

This session was chaired by Prof Gosden and aimed at uncovering current consensus, or lack thereof, on the 
technical strategies in place to achieve cryopreservation of oocytes, embryos, and ovarian tissue. Emphasis was placed on three factors: efficacy, safety, and cost. And the focus of the presentations and discussion was appropriately confined to whether slow freeze or vitrification was the more efficient of the technologies proposed. In the end, as Gosden declared, with only two options at hand, there was widespread acceptance that at present both techniques offer safe and variably efficient means for the cryopreservation of all materials until further data from controlled studies can offer information to the contrary. With respect to the issues of cost, it was generally recognized that because the investment for slow freeze cryopreservation, as well as technical training of embryologists, has been realized in many established clinics, this should remain the approach of choice given acceptable standards of clinical success. In newly established clinics, vitrification methods are recommended for adoption given efforts to minimize patient costs, the relative ease of procedures, and potential better outcomes. There was in addition a call to form a data registry for outcomes for each procedure and application. However, the fact that many variables remain with respect to cell/tissue preparation, formulations for both cryoprotectant and thawing protocols, and even the requirements for the handling of cells/tissues following thawing, was viewed as deterrent to providing general guidelines for implementation until more systematic and controlled studies can be performed. This was considered as an impetus for future funding that will benefit not only fertility preservation applications but also human ARTs in general. 